\title{
Datenschutzrechtliche Informationen im Zusammenhang mit der Corona-Pandemie
}

Die unabhängigen Datenschutzaufsichtsbehörden des Bundes und der Länder erreichen vermehrt Anfragen von Arbeitgebern/ Dienstherren, ob und wie personenbezogene Daten von Mitarbeitern sowie Gästen und Besuchern bei im Zusammenhang mit der Corona-Pandemie stehenden Maßnahmen verarbeitet werden können. Dazu einige allgemeine Hinweise:

Werden im Zusammenhang mit der Corona-Pandemie personenbezogene Daten erhoben, werden in den meisten Fällen Bezüge zwischen Personen und deren Gesundheitszustand hergestellt. Ab diesem Zeitpunkt handelt es sich um Gesundheitsdaten, die nach Artikel 9 Datenschutz-Grundverordnung (DSGVO) besonders geschützt sind. Auch wenn eine Verarbeitung von $\mathrm{Ge}$ sundheitsdaten grundsätzlich nur restriktiv möglich ist, können für verschiedene Maßnahmen zur Eindämmung der Corona-Pandemie oder zum Schutz von Mitarbeiterinnen und Mitarbeitern datenschutzkonform Daten erhoben und verwendet werden. Dabei ist der Grundsatz der Verhältnismäßigkeit und der gesetzlichen Grundlage stets zu beachten. Beispielsweise können die folgenden Maßnahmen zur Eindämmung und Bekämpfung der CoronaPandemie als datenschutzrechtlich legitimiert betrachtet werden:

- Erhebung und Verarbeitung personenbezogener Daten (einschließlich Gesundheitsdaten) von Beschäftigten durch den Arbeitgeber oder Dienstherren um eine Ausbreitung des Virus unter den Beschäftigten bestmöglich zu verhindern oder einzudämmen. Hierzu zählen insbesondere Informationen zu den Fällen:

- in denen eine Infektion festgestellt wurde oder Kontakt mit einer nachweislich infizierten Person bestanden hat.

- in denen im relevanten Zeitraum ein Aufenthalt in einem vom Robert-Koch-Institut (RKI) als Risikogebiet eingestuften Gebiet stattgefunden hat.

- Erhebung und Verarbeitung personenbezogener Daten (einschließlich Gesundheitsdaten) von Gästen und Besuchern, insbesondere um festzustellen, ob diese

- selbst infiziert sind oder im Kontakt mit einer nachweislich infizierten Person standen.

- sich im relevanten Zeitraum in einem vom RKI als Risikogebiet eingestuften Gebiet aufgehalten haben.

- Die Offenlegung personenbezogener Daten von nachweislich infizierten oder unter Infektionsverdacht stehenden Personen zur Information von Kontaktpersonen ist demgegenüber nur rechtmäßig, wenn die Kenntnis der Identität für die Vorsorgemaßnahmen der Kontaktpersonen ausnahmsweise erforderlich ist.

\section{Rechtliche Hintergrundinformationen:}

Die vorstehenden Maßnahmen lassen sich rechtlich auf Grundlage der DSGVO und des BDSG (ggf. in Verbindung mit Landesdatenschutz- und weiteren Fachgesetzen) legitimieren. Je nach Maßnahme können die einschlägigen Rechtsgrundlagen dabei leicht variieren. Ungeachtet dessen gelten aber die folgenden allgemeinen Grundsätze:

Die Berechtigung zur Verarbeitung personenbezogener Mitarbeiterdaten ergibt sich in diesen Fällen für öffentlich-rechtliche
Arbeitgeber grundsätzlich aus Art. 6 Abs. 1 Satz 1 lit. e) DSGVO und für Arbeitgeber im nicht-öffentlichen Bereich aus $\$ 26$ Abs 1 BDSG bzw. Art. 6 Abs. 1 Satz 1 lit. f) DSGVO jeweils i.V.m. den einschlägigen beamtenrechtlichen sowie tarif-, arbeits- und sozialrechtlichen Regelungen des nationalen Rechts. Soweit Gesundheitsdaten verarbeitet werden, sind zudem auch $\$ 26$ Abs. 3 BDSG und Art. 9 Abs. 2 lit. b) DSGVO einschlägig. Bei Art. 9 Abs. 2 lit. b) DSGVO umfasst der Begriff „Arbeitsrecht“ nach Auffassung der Datenschutzaufsichtsbehörden auch das deutsche Beamtenrecht. Zugunsten des öffentlich-rechtlichen Arbeitgebers könnte zusätzlich Art. 9 Abs. 2 lit. g) DSGVO herangezogen werden, da die Fürsorgepflicht im Sinne der Gesundheitsvorsorge hier auch einem wichtigen öffentlichen Interesse dient.

Maßnahmen gegenüber Dritten können bei öffentlichen Stellen auf Art. 6 Abs. 1 Satz 1 lit. c) und e) ggf. in Verbindung mit den jeweiligen Landesdatenschutzgesetzen gestützt werden. Im nichtöffentlichen Bereich kann Art. 6 Abs. 1 Satz 1 lit. f) DS-GVO als Rechtsgrundlage herangezogen werden. Soweit besonders sensible Daten - wie Gesundheitsdaten - betroffen sind, findet zudem Art. 9 Abs. 2 lit. i) i.V.m. $\$ 22$ Abs. 1 Nr. 1 lit. c) BDSG Anwendung.

Die Fürsorgepflicht der Arbeitgeber bzw. der Dienstherren verpflichtet diese den Gesundheitsschutz der Gesamtheit ihrer Beschäftigten sicherzustellen. Hierzu zählt nach Ansicht der unabhängigen Datenschutzaufsichtsbehörden auch die angemessene Reaktion auf die epidemische bzw. inzwischen pandemische Verbreitung einer meldepflichtigen Krankheit, die insbesondere der Vorsorge und im Fall der Fälle der Nachverfolgbarkeit (also im Grunde nachgelagerte Vorsorge gegenüber den Kontaktpersonen) dient. Diese Maßnahmen müssen dabei natürlich immer auch verhältnismäßig sein. Die Daten müssen vertraulich behandelt und ausschließlich zweckgebunden verwendet werden. Nach Wegfall des jeweiligen Verarbeitungszwecks (regelmäßig also spätestens dem Ende der Pandemie) müssen die erhobenen Daten unverzüglich gelöscht werden.

Eine Einwilligung der von Maßnahmen Betroffenen allein sollte hingegen vorliegend nur als datenschutzrechtliche Verarbeitungsgrundlage in Betracht gezogen werden, wenn die Betroffenen über die Datenverarbeitung informiert sind und freiwillig in die Maßnahme einwilligen können.

Zusätzlich zu den bestehenden Rechtsgrundlagen für die Datenverarbeitung auf Seiten des Arbeitgebers ergeben sich aus dem Beamtenrecht, aus dem Tarifrecht bzw. dem Arbeitsrecht für Beschäftigte verschiedene Nebenpflichten, unter anderem auch Rücksichts-, Verhaltens- und Mitwirkungspflichten gegenüber ihrem Arbeitgeber und Dritten. Vorliegend stellt nach Auffassung der Datenschutzaufsichtsbehörden beispielsweise die Pflicht zur Information des Dienstherrn bzw. des Arbeitgebers über das Vorliegen einer Infektion mit dem Corona-Virus eine solche Nebenpflicht zum Schutz hochrangiger Interessen Dritter dar, aus der unter gewissen Voraussetzungen auch eine Offenlegungsbefugnis gemäß Art. 6 Abs. 1 lit. c) und f) DSGVO bezüglich personenbezogener Daten der Kontaktpersonen folgt. 